\title{
Amplitude noise reduction in semiconductor lasers with weak, dispersive optical feedback
}

\author{
J. Kitching, R. Boyd, and A. Yariv \\ Department of Applied Physics, California Institute of Technology, 128-95, Pasadena, California 91125
}

Y. Shevy

Department of Physics, University of Miami, P.O. Box 248046, Coral Gables, Florida 33124

Received March 18, 1994

\begin{abstract}
We present the theory and measurements of the amplitude noise spectrum from a semiconductor laser with weak optical feedback $\left(P_{\mathrm{fb}} / P_{\text {out }} \approx 10^{-6}\right)$ from an external cavity containing an element of dispersive loss. The laser noise is found to be reduced over most of the low-frequency spectrum, although an increase in the noise is observed at frequencies corresponding to multiples of the external-cavity free spectral range. The low-frequency noise reduction closely follows theoretical predictions, and a reduction of as much as $7 \mathrm{~dB}$ is measured at an injection current of 1.5 times the threshold current. The potential of this method for contributing to the production of amplitude-squeezed light is discussed.
\end{abstract}

The effects of optical feedback on the dynamic and noise properties of semiconductor lasers have been under investigation for some time. ${ }^{1}$ Large reductions in the laser linewidth have been obtained by taking advantage of the correlation between the amplitude and phase noise caused by phase-toamplitude coupling in semiconductor lasers., ${ }^{2,3}$ Under different conditions this correlation can also be used to reduce the laser amplitude noise. ${ }^{4}$ Although amplitude noise reduction by use of weak optical feedback has been both predicted ${ }^{4}$ and observed, ${ }^{2}$ a thorough understanding of this subject is still lacking. It has been reported ${ }^{5}$ that optical feedback tends to increase the noise rather than reduce it, and the conditions under which noise reduction is possible remain unclear. The goal of this Letter is to provide a comparison of experimental results with the theory of amplitude noise reduction by use of optical feedback and to investigate this phenomenon in some detail.

In the modeling of the amplitude noise of a semiconductor laser above threshold it is necessary to start from quantum-mechanical rate equations. We follow the Langevin equation approach ${ }^{6,7}$ but include in the field equation a term that represents feedback from an external cavity. ${ }^{1}$ We permit this feedback term to describe a dispersive element (such as an atomic vapor) that may be present in the cavity.

The equations of motion for the slowly varying Heisenberg operators for the internal field $\hat{A}(t)$, carrier density $N_{c}(t)$, and external field $\hat{r}(t)$ are written as

$$
\begin{aligned}
\frac{\mathrm{d} \hat{A}(t)}{\mathrm{d} t}= & -\frac{1}{2}\left[\frac{1}{\tau_{p}}+2 i\left(\omega_{L}-\omega_{0}\right)-\frac{\omega}{\mu^{2}}\left(\chi_{i}-i \chi_{r}\right)\right] \\
& \times \hat{A}(t)+\hat{G}(t)+\hat{g}(t)+\left(\frac{1}{\tau_{\mathrm{pe}}}\right)^{1 / 2} f_{e}(t) \\
& +\kappa \exp \left(i \phi_{m}\right) \hat{r}(t-\tau),
\end{aligned}
$$

$$
\begin{aligned}
\frac{\mathrm{d} N_{c}(t)}{\mathrm{d} t}= & P-\frac{N_{c}(t)}{\tau_{\mathrm{sp}}}-\frac{\omega}{\mu^{2}} \chi_{i} \hat{A}^{\dagger} \hat{A}-\left\langle\frac{\omega}{\mu^{2}} \chi_{i}\right\rangle \\
& +\Gamma_{p}(t)+\Gamma_{\mathrm{sp}}(t)+\Gamma(t), \\
& \hat{r}(t)=-\hat{f}_{e}(t)+\sqrt{1 / \tau_{\mathrm{pe}}} \hat{A}(t),
\end{aligned}
$$

where $\tau_{p}$ is the total photon lifetime, $\tau_{\mathrm{pe}}$ is the photon lifetime that is due to facet losses only, $\omega_{L}$ is the lasing frequency, $\omega_{0}$ is the cold semiconductor cavity resonant frequency, $\mu$ is the nonresonant refractive index, $\chi$ is the resonant optical susceptibility, $P$ is the pump rate, and $\tau_{\mathrm{sp}}$ is the spontaneous emission carrier lifetime. Langevin noise operators $\hat{G}(t), \hat{g}(t)$, and $\hat{f}_{e}(t)$, respectively, correspond to dipole moment fluctuations, internal optical losses, and incident vacuum fluctuations. $\Gamma_{p}(t), \quad \Gamma_{\mathrm{sp}}(t)$, and $\Gamma(t)$, respectively, correspond to pump noise, carrier noise that is due to spontaneous emission into nonlasing modes, and dipole moment fluctuations. The correlation relations for these noise operators are identical to those given in Ref. 7. The last term in Eq. (1) is the feedback term, where $\kappa=\sqrt{P_{\mathrm{fb}} / P_{\text {out }}} / \tau_{\mathrm{pe}}$ is the frequencyindependent feedback coupling rate, $P_{\mathrm{fb}}$ is the feedback power, $P_{\text {out }}$ is the output power, $\phi_{m}$ is the end-mirror reflectivity, and $\tau=\tau_{0}+\mathrm{d} \phi_{r} / \mathrm{d} \omega$ is the total delay through the external cavity, which includes both the nonresonant delay $\tau_{0}$ and the delay that is due to the dispersion of the atomic vapor $\mathrm{d} \phi_{r} / \mathrm{d} \omega$.

The above equations are linearized, and coupled small-signal equations are then derived for the internal field amplitude, phase, and carrier-density fluctuation operators. Solving the Fourier-transformed algebraic equations, we obtain the power spectral density for the external field amplitude noise, normalized to the standard quantum limit (SQL): 


$$
\begin{aligned}
P_{\Delta r}(\Omega)= & \frac{4 A_{3}{ }^{2}\left|1+C_{i}+\alpha C_{r}\right|^{2}}{\tau_{\mathrm{pe}}|D|^{2}}\left(\frac{4 k T}{q^{2} R_{s}}+\frac{N_{c 0}}{\tau_{\mathrm{sp}}}\right)+\frac{\left(\Omega^{2}+A_{1}{ }^{2}\right)\left|1+C_{i}\right|^{2}}{\tau_{\mathrm{pe}} \tau_{p 0}|D|^{2}}+\frac{2 n_{\mathrm{sp}}\left(\Omega^{2}+A_{1}{ }^{2}\right)}{\tau_{p} \tau_{\mathrm{pe}}|D|^{2}}\left|C_{r}\right|^{2} \\
& +\left|1-\frac{\left(i \Omega-A_{1}\right)\left(1+C_{i}\right)}{\tau_{\mathrm{pe}} D}\right|^{2}+\frac{\left(2 n_{\mathrm{sp}}-1\right)\left|1+C_{i}\right|^{2}}{\tau_{p} \tau_{\mathrm{pe}}|D|^{2}}\left|\left(i \Omega-A_{1}\right)-2 A_{0} A_{3}\left(1+\alpha \frac{C_{r}}{1+C_{i}}\right)\right|^{2} .
\end{aligned}
$$

The coefficients in Eq. (4) are given by

$$
\begin{aligned}
C_{i}(\Omega)= & \{[1-\exp (-i \Omega \tau)] /(i \Omega \tau)\} \kappa_{0} \tau \cos \left(\phi_{0}\right), \\
C_{r}(\Omega)= & -\{[1-\exp (-i \Omega \tau)] /(i \Omega \tau)\} \kappa_{0} \tau \sin \left(\phi_{0}\right), \\
D(\Omega)= & i \Omega\left(i \Omega-A_{1}\right)\left[\left(1+C_{i}\right)^{2}+C_{r}^{2}\right] \\
& -A_{2} A_{3}\left(1+C_{i}+\alpha C_{r}\right), \\
A_{1}= & -\left(1 / \tau_{\mathrm{sp}}+1 / \tau_{\mathrm{st}}\right), \\
A_{2}= & -2 A_{0} E_{\mathrm{cv}} / n_{\mathrm{sp}} \\
A_{3}= & 1 /\left(2 A_{0} \tau_{\mathrm{st}}\right)
\end{aligned}
$$

where $\tau_{\mathrm{st}}$ is the carrier stimulated-emission lifetime, $E_{\mathrm{cv}} / n_{\mathrm{sp}}=\omega\left\langle\chi_{i}\right\rangle / \mu^{2}$ is the net gain, and $\phi_{0}=$ $\phi_{m}+\omega_{L} \tau+\phi_{r}$ is the total phase change of the field through the external cavity.

A detailed analysis of Eq. (4) yields several important consequences at frequencies below the relaxation resonance. First, pump noise and noise that is due to spontaneous emission into nonlasing modes (the dominant noise mechanisms at high pump rates) are unaffected by the optical feedback. Thus it is clear that, if amplitude-squeezed light is to be obtained or enhanced, the feedback must be used in conjunction with pump suppression. ${ }^{7}$ Second, noise that is due to vacuum fluctuations (spontaneous emission into the lasing mode) and dipole moment fluctuations, which are the dominant noise sources at low pump rates, can be reduced by a maximum of $1+\alpha^{2}$ in accordance with the semiclassical theory. ${ }^{4}$ Thus we expect to obtain large reductions in the amplitude noise at injection currents near threshold, with the reduction decreasing at higher injection currents. Such behavior is shown in Fig. 1. We also find that an enhancement of the squeezing in a pumpsuppressed semiconductor laser occurs at moderate pump rates (see Fig. 1, inset). This enhancement is potentially important when the laser is at room temperature, at which the high injection currents needed to produce more complete squeezing cannot be obtained.

The experimental setup, shown in Fig. 2, was described previously. ${ }^{3}$ A single-mode GaAs semiconductor laser (STC LT50A-03U) lasing at $852 \mathrm{~nm}$ and with a threshold current of $51.4 \mathrm{~mA}$ was coupled to a $75-\mathrm{cm}$ external cavity containing a $\mathrm{Cs}$ cell placed between two crossed polarizers. The Cs was heated to $90^{\circ} \mathrm{C}$, and an axial magnetic field of $150 \mathrm{G}$ was applied. Faraday rotation caused a small fraction $\left(10^{-0}\right)$ of the output power to be reflected back into the laser when the laser was tuned to the Dopplerbroadened $D_{2}$ line at $852 \mathrm{~nm}$. Because of the wavelength selectivity of the Cs the feedback affected the dynamics of only a single longitudinal mode, a factor we have found to be important when using these lasers in achieving a reduction in the laser amplitude noise with optical feedback. Most of the light (98\%) was coupled out of the external cavity (at BS1) and was then passed through a pair of optical isolators that provided $60 \mathrm{~dB}$ of isolation. For measurement of the low-frequency noise the beam was shone onto a broad-area high-efficiency photodiode (Hamamatsu .1722-01), and the resulting photocurrent was amplified and sent to an electronic spectrum analyzer. The net current-to-current quantum efficiency above threshold with this detector was only $36 \%$. The data were normalized to the SQL, which we calibrated by shining a filtered incandescent lamp onto the detector with enough intensity to produce an equal photocurrent. The detector was found to be linear at dc to photocurrents as high as $20 \mathrm{~mA}$ but showed some saturation at frequencies greater than $100 \mathrm{MHz}$.

The laser was locked to the top of the Cs Faraday line, and the amplitude noise spectrum was mea-

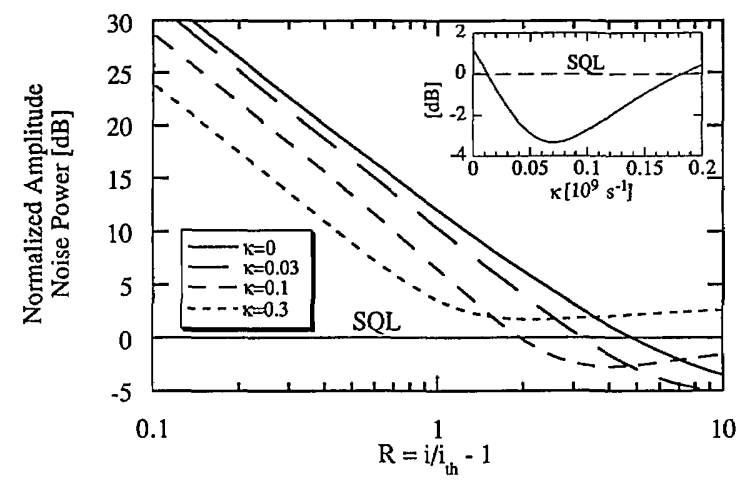

Fig. 1. Theoretical prediction of the noise reduction at $116 \mathrm{MHz}$ for different feedback coupling rates (in units of $10^{9} \mathrm{~s}^{-1}$ ) in a pump-suppressed semiconductor laser. The inset shows the squeezing enhancement at a pump rate of $R=i_{l} / i_{\text {th }}-1=4$. The parameter values used are $\tau_{p}=\tau_{\mathrm{pe}}=9.1 \times 10^{-12} \mathrm{~s}, \tau_{\mathrm{sp}}=4.3 \times 10^{-9} \mathrm{~s}, n_{\mathrm{sp}}=1.25$, $\beta=10^{-5}, \alpha=-3.4, \tau=1.4 \times 10^{-8} \mathrm{~s}, \eta_{\text {int }}=0.85$, and $\eta_{\mathrm{ext}}=0.9$.

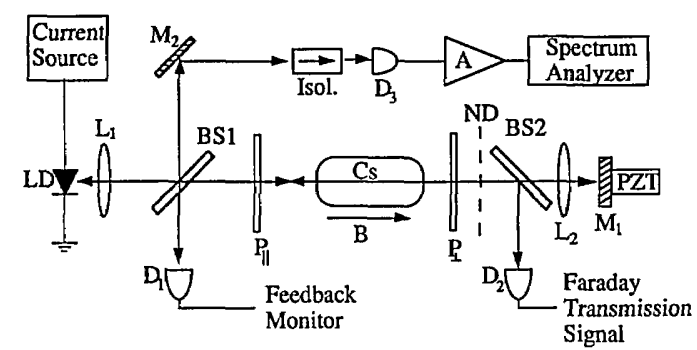

Fig. 2. Experimental setup: LD, laser diode; L's, lenses; BS's, beam splitters; M's, mirrors; P's, polarizers; D's, detectors; ND, neutral-density filter; PZT, piezoelectric transducer; Isol., isolator; A, amplifier; B, magnetic field. The amplitude noise power is measured at $\mathrm{D}_{3}$, and the feedback power is monitored at $D_{1}$. 


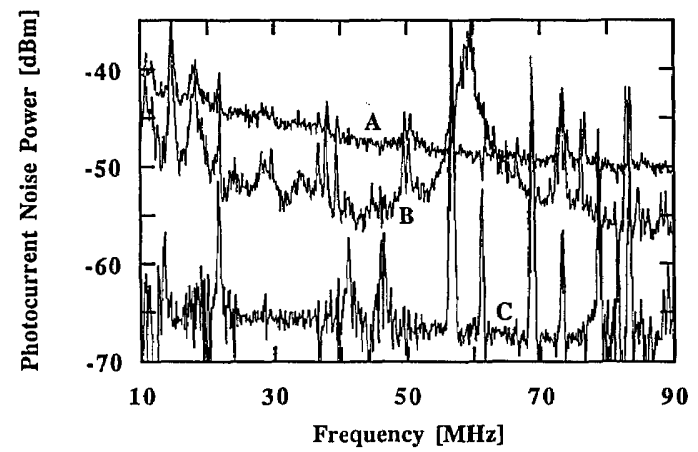

Fig. 3. Frequency dependence of the amplitude noise power at an injection current of $76 \mathrm{~mA}$ for free-running (trace A), $P_{\mathrm{fb}} / P_{\text {out }}=4 \times 10^{-7}$ (trace B), and SQL (trace C) conditions.

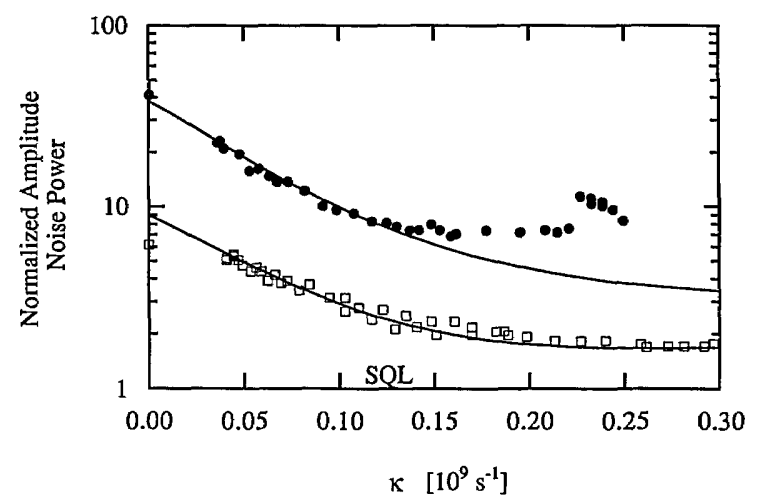

Fig. 4. Amplitude noise power at $116 \mathrm{MHz}$, normalized to the SQL, as a function of feedback power at injection currents of $76 \mathrm{~mA}$ (filled circles) and $97 \mathrm{~mA}$ (open squares). The solid curves are the theoretical predictions based on Eq. (4).

sured. A portion of this spectrum is shown in Fig. 3, taken at an injection current of $76 \mathrm{~mA}$. Under feedback conditions the amplitude noise is seen to be reduced at most frequencies, with peaks occurring at multiples of $60 \mathrm{MHz}$, a frequency corresponding to the external-cavity free spectral range modified from its empty-cavity value of $200 \mathrm{MHz}$ by the dispersion of the Cs.

The reduction of the amplitude noise at a fixed frequency of $116 \mathrm{MHz}$ was measured as a function of feedback power; the results are plotted in Fig. 4. For each measurement the feedback phase was adjusted so that the laser frequency with feedback was the same as the unperturbed laser frequency. ${ }^{3}$ This not only fixed the phase to a constant known value but also avoided simultaneous locking to more than one external-cavity mode. Each measurement was normalized to the SQL after subtraction of the background amplifier noise. At an injection current of $76 \mathrm{~mA}$ (filled circles) the maximum noise reduction was found to be $7 \mathrm{~dB}$; at higher injection currents this maximum reduction decreased (open squares). We also plotted the theoretical predictions (solid curves), using the parameter values given in Fig. 1 except for the following: $n_{\mathrm{sp}}=1$ (76 mA), $\alpha=-4.5(76 \mathrm{~mA})$, and $\eta_{\text {ext }}=0.42$. A reasonable agreement between theory and experiment is found. At the lower injection current the laser noise was found to increase if the feedback cou- pling rate was increased to greater than $\sim 0.2 \times$ $10^{9} \mathrm{~s}^{-1}$, causing a deviation from the theoretical prediction. It is possible that this increase is caused by the onset of coherence collapse or by increased power in the laser side modes.

The difficulty of tuning the laser to the Cs line and the danger of thermal damage to the laser at high output powers limited the range of injection currents within which these measurements could be made. Because of the high threshold current of our laser the maximum pump rate obtainable was $R=$ $i / i_{\text {th }}-1=1.4$, which permitted a noise reduction to within $2.3 \mathrm{~dB}$ of the SQL but which was not enough to produce squeezed light. Since our laser was operated with a current source, the pump noise should have been suppressed and the noise reduction then limited, at maximum feedback levels, by carrier noise that is due to spontaneous emission into nonlasing modes. As Fig. 1 shows, a pump rate of $\sim R=3$ is required before significant low-frequency squeezing should be observable. It was reported recently ${ }^{8}$ that a reduction of the amplitude noise of a semiconductor laser to below the SQL was obtained by use of strong feedback from a grating. We intend to repeat our weak-feedback experiment, using a laser with a lower threshold current and a balanced homodyne detector to quantitatively measure the effects of the feedback on the laser noise in the quantum regime.

The effects of optical feedback on the amplitude noise spectrum of a semiconductor laser have been examined theoretically and experimentally. Under weak-feedback conditions $\left(P_{\mathrm{fb}} / P_{\text {out }} \approx 10^{-6}\right)$ the lowfrequency amplitude noise is reduced by as much as $7 \mathrm{~dB}$ at moderate injection currents. The dependence of this reduction on the feedback power compares favorably with theoretical predictions based on a quantum-mechanical model of the laser noise. At moderate pump rates, achievable at room temperature, an enhancement of the low-frequency amplitude squeezing in a pump-suppressed semiconductor laser is predicted.

The authors gratefully acknowledge support from the National Science Foundation, the Advanced Research Projects Agency, and the U.S. Office of Naval Research.

\section{References}

1. R. Lang and K. Kobayashi, IEEE J. Quantum Electron. QE-16, 347 (1980).

2. B. Dahmani, L. Hollberg, and R. Drullinger, Opt. Lett. 12, 876 (1987).

3. Y. Shevy, J. Iannelli, J. Kitching, and A. Yariv, Opt. Lett. 17, 661 (1992).

4. A. Yariv, R. Nabiev, and K. Vahala, Opt. Lett. 15, 1359 (1990); R. Nabiev, Y. Popov, and A. Yariv, J. Phys. III 2, 1605 (1992).

5. W. H. Richardson and R. M. Shelby, Phys. Rev. Lett. 64, 400 (1990).

6. H. Haug, Phys. Rev. 184, 338 (1969).

7. Y. Yamamoto, S. Machida, and O. Nilsson, Phys. Rev. A 34, 4025 (1986).

8. M. J. Freeman, H. Wang, D. G. Steel, R. Craig, and D. R. Scifres, Opt. Lett. 18, 2141 (1993). 